\title{
Exámenes preoperatorios en cirugías electivas no cardiacas de riesgo bajo e intermedio: revisión sistemática y análisis costo-efectividad
}

Edinson Dante Meregildo-Rodriguez*1,2,a

\section{RESUMEN}

Los exámenes preoperatorios son parte habitual en la práctica clínica. Sin embargo, su requerimiento no se sustenta siempre en la mejor evidencia científica. Cuando se solicitan sin justificación pueden generar resultados falsos positivos, la necesidad de repetir o solicitar nuevos exámenes e interconsultas, prolongar la estancia hospitalaria, ocasionar problemas al paciente (mayor riesgo, confusión y ansiedad) e incremento de los costos. Se realizó una revisión sistemática y análisis costo-efectividad de los exámenes preoperatorios en las cirugías electivas no cardíacas de riesgo bajo e intermedio más frecuentes realizadas en el Hospital Regional Lambayeque. Según el análisis costo-efectividad, el monto anual de los exámenes preoperatorios innecesarios sería de S/ 62 778, pero si se solicitaran de manera adecuada y se siguieran las pautas establecidas en la Guía NICE, este costo se reduciría en 38,34 \% (es decir S/ 138,40 por cada paciente). Los resultados muestran que no se debe solicitar exámenes preoperatorios de manera rutinaria para las cirugías no cardíacas electivas de riesgo bajo e intermedio y que una minuciosa evaluación médica preoperatoria reduciría, de manera segura, la necesidad otras pruebas para este tipo de cirugías.

Palabras clave: Periodo preoperatorio; Procedimientos quirúrgicos; Guía de práctica clínica; Prueba de laboratorio (Fuente: DeCS BIREME).

\section{Preoperative testing for low- and intermediate-risk non-cardiac elective surgeries: a systematic review and cost-effectiveness analysis}

\section{ABSTRACT}

Preoperative tests are very common in daily clinical practice. However, ordering preoperative tests is not generally based on the best scientific evidence. When preoperative tests are ordered without justification, they may generate false-positive results, cause to repeat or order new tests and consultations, prolong hospital stay, result in problems to the patient (more risks, confusion and anxiety), and increase costs. A systematic review and cost-effectiveness analysis of preoperative tests for the most frequent low- and intermediate-risk non-cardiac elective surgeries performed at the Hospital Regional Lambayeque were conducted. According to the cost-effectiveness analysis, the annual cost of unnecessary preoperative tests amounts to S/ 62,778.00. However, if preoperative tests were adequately ordered and NICE guidelines were followed, their cost would decrease in $38.34 \%$ (i.e., S/ 138.40 for each patient). The results show that preoperative tests should not be ordered for low- and intermediate-risk non-cardiac elective surgeries on a routinely basis. A thorough preoperative medical evaluation would safely decrease the need of other tests for this type of surgery.

Keywords: Preoperative period; Surgical procedures, operative; Practice guideline; Laboratory test (Source: MeSH NLM).

1 Universidad César Vallejo, Escuela de Medicina. Trujillo, Perú.

2 Hospital Regional Lambayeque, Servicio de Medicina Interna. Lambayeque, Perú.

a Médico Internista, Maestro en Medicina, Doctor en Investigación Clínica y Traslacional.

* Autor corresponsal. 


\section{INTRODUCCIÓN}

Los exámenes preoperatorios (EPO) forman parte habitual de la práctica clínico-quirúrgica. Existen diversos argumentos que intentan justificar el empleo de estas pruebas; por ejemplo, identificar alteraciones en los EPO que influirían en la aparición de complicaciones, establecer valores basales que podrían vigilarse y modificarse después de la cirugía y también motivos médico-legales ${ }^{(1-8)}$.

A pesar de que los EPO son rutinarios, su solicitud no siempre está basada en evidencia sólida. Se podría definir como EPO innecesario al que no está recomendado por una guía de práctica clínica y cuyo resultado es 1) normal o 2 ) anormal, pero que no se traduce en ningún cambio en el manejo ${ }^{(9-23)}$.

La realización indiscriminada e injustificada de exámenes preoperatorios puede generar resultados de laboratorio anormales, ocasionar repetición de exámenes previos, ordenar nuevos análisis cada vez más invasivos (y potencialmente nocivos) y ocasionar confusión y ansiedad en el paciente. Además, los resultados falsos-positivos podrían retrasar las cirugías. Los EPO innecesarios representan una carga económica importante si se consideran la pérdida de horas laborales y el desperdicio de recursos (24-31). Tras realizar una búsqueda sistemática de la literatura publicada, no encontramos ningún estudio en nuestro país que haya realizado un análisis costo-efectividad de los EPO en cirugías electivas $(10-13,15,16,18-20,26,29,31,37-40)$. El objetivo de esta investigación es realizar una revisión sistemática de la literatura y efectuar un análisis costo-efectividad de los EPO en cirugía electivas no cardiacas de riesgo bajo e intermedio.

\section{Estrategia de búsqueda}

Se realizó una revisión sistemática en las bases de datos Pubmed, EBSCO, Scielo, Google Scholar, Medline, y Latindex, empleando palabras clave: "cost-effectiveness", "cost-benefit", "preoperative test", "diagnostic test", "usefulness", y "elective surgery"; y combinaciones de estos términos. Se seleccionaron solo artículos originales en texto completo publicados desde 1990 al 2020. Se excluyeron los siguientes artículos: los que presentaban solo el abstract, los que trataban de cirugías de emergencia, cardiacas y de alto riesgo, o que incluyeran solamente a niños. La búsqueda se realizó entre el 1 al 20 de enero 2020.

\section{Análisis costo-efectividad}

Para realizar un análisis costo-efectividad (ACE) se define una proporción que considera como numerador el costo promedio de los EPO por paciente, y en el denominador, la proporción de efectividad clínica (en términos de número de anormalidades significativas). Se compara la intervención habitual en la práctica clínica actual (intervención "A") con la nueva forma de solicitar exámenes preoperatorios de acuerdo a la guía NICE (intervención "B"). A continuación, se calcula la relación entre los costos y los efectos de ambas intervenciones. Como se detalla en la ecuación y tabla 2, se calculan el cociente entre el costo neto de la intervención sobre el beneficio neto o efectividad [costo-efectividad medio (CEM)] y el cociente entre el incremento del costo $(\Delta C)$ sobre el incremento de la efectividad $(\triangle \mathrm{E})$ [costo-efectividad incremental (CEI)]. Entre la comparación de ambos cocientes, se decide cuál es la intervención con mayor costo-efectividad ${ }^{(24,25)}$.

Se encontró 22 artículos originales que reunieron los criterios de búsqueda. De estos, se excluyeron cuatro por no tener disponible la versión extensa y uno por evaluar solamente a niños. Por tanto, para el análisis final se incluyeron 17 estudios originales $(10-13,15,16,18-20,26,29,31,37-40)$ (Tabla 1). De este grupo, cinco provenían de Latinoamérica ${ }^{(10,12,13,31,37)}$, y de ellos, solamente tres incluyeron algún tipo de análisis de $\operatorname{costos}^{(12,13,31)}$. De estas cinco investigaciones, dos utilizaron variables y diseños muy similares (corte transversal) e incluyeron análisis de costos, por lo que resultaron de particular interés ${ }^{(12,13)}$. El estudio de Morales-Orozco C (México) también analiza costos, pero tiene las siguientes limitaciones: es retrospectivo y fue realizado hace 15 años, por lo que no fue considerado finalmente ${ }^{(31)}$. León-Jiménez et al. ${ }^{(12)}$ revisaron 378 cirugías electivas realizadas en el Hospital Regional Lambayeque (HRL) y seleccionaron aleatoriamente 100 casos de pacientes sin comorbilidades y eligieron las cirugías más frecuentes.

La finalidad del estudio fue estimar la frecuencia y costo de los EPO innecesarios. Definieron como exámenes innecesarios aquellos que no son recomendados por la guía NICE sobre exámenes preoperatorios del Reino Unido (32). Namoncura et al. ${ }^{(13)}$ incluyeron pacientes de características similares al estudio previo, pero buscaron evaluar utilidad y costo-efectividad de los EPO en cirugías electivas otorrinolaringológicas. Analizaron 160 historias clínicas de pacientes ASA I operados en un hospital de Chile. 
Exámenes preoperatorios en cirugías electivas no cardiacas de riesgo bajo e intermedio: revisión sistemática y análisis costo-efectividad

Tabla 1. Estudios sobre exámenes preoperatorios (EPO) en cirugías no cardiacas de riesgo bajo intermedio desde 1990 hasta la actualidad

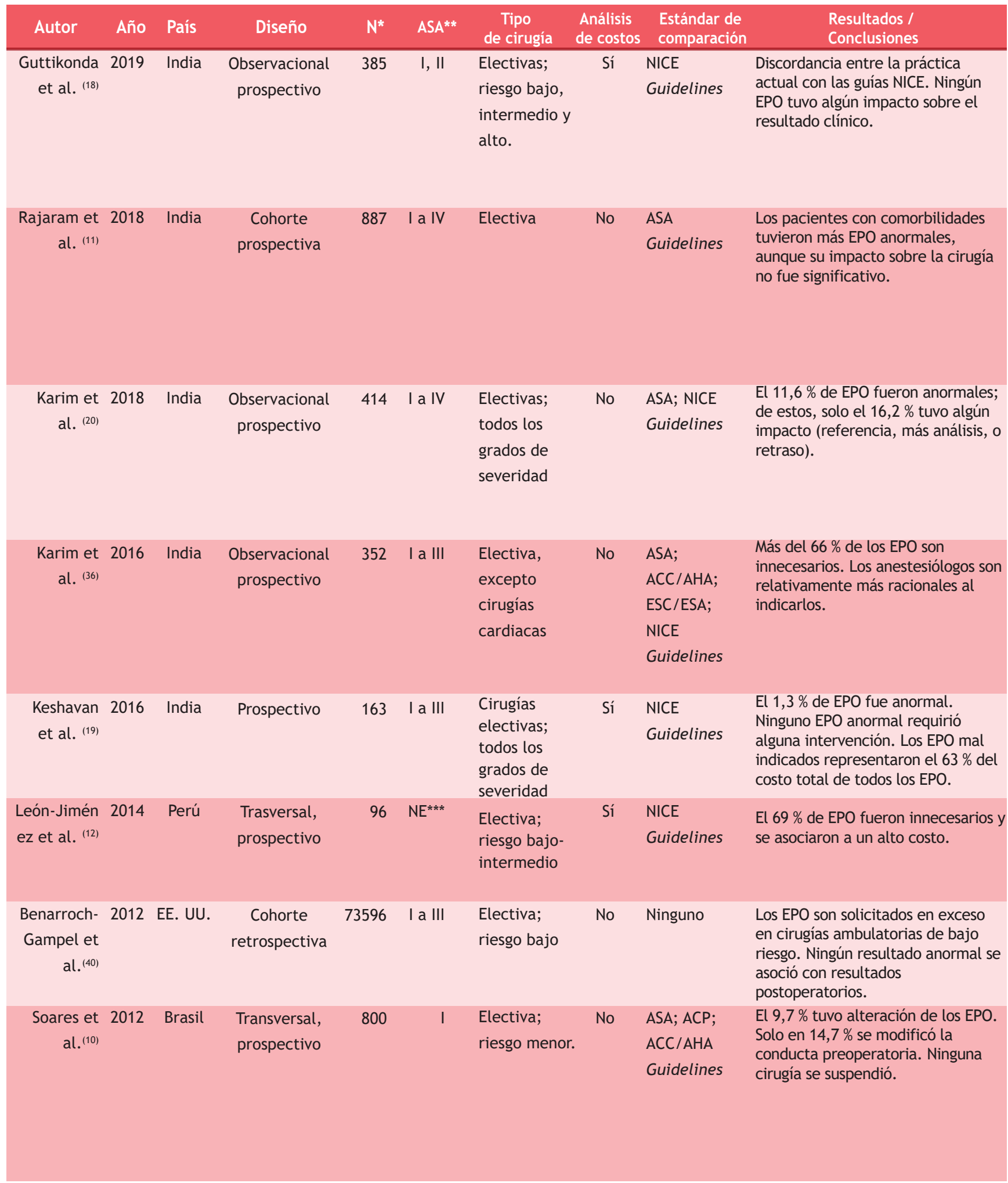




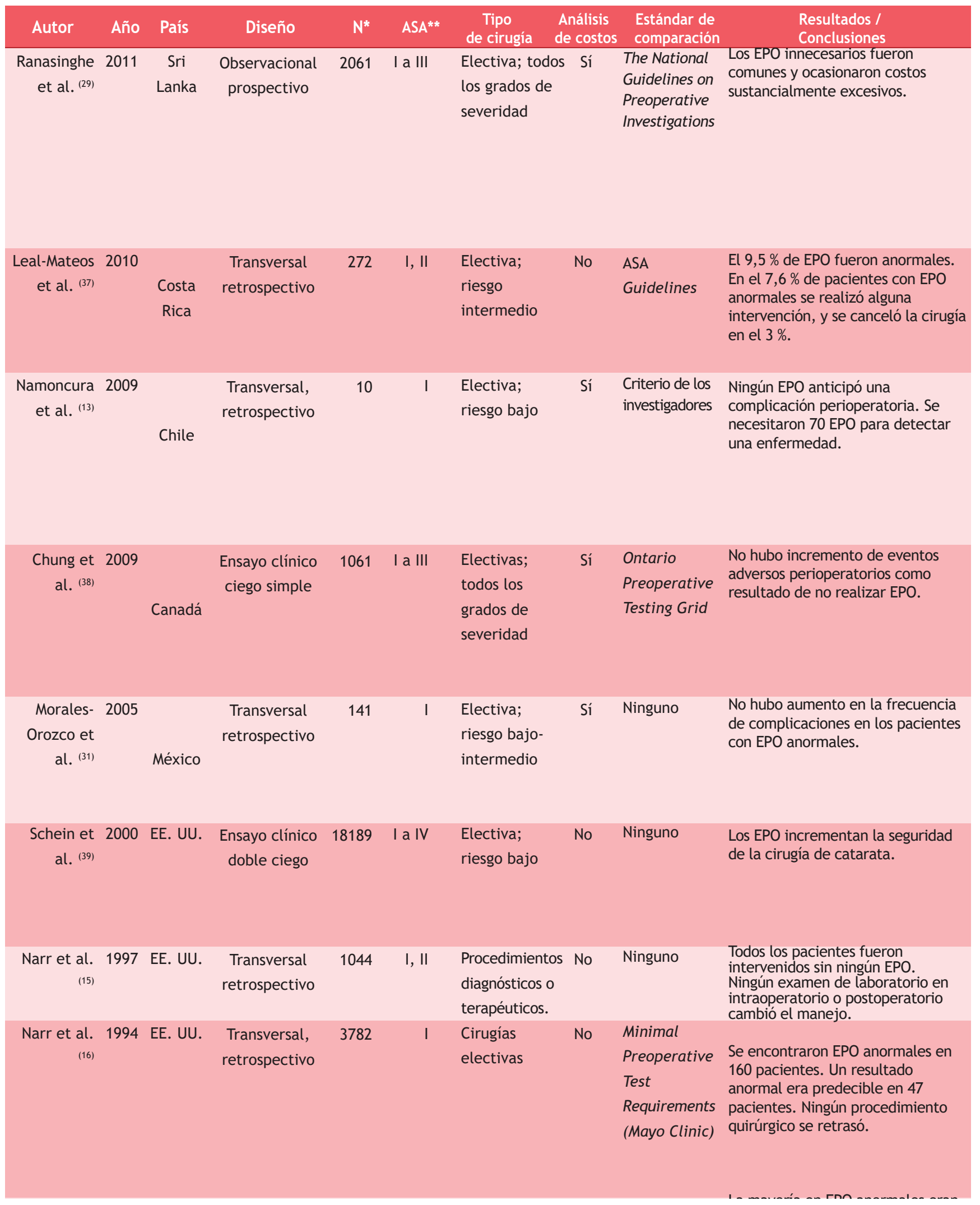


Exámenes preoperatorios en cirugías electivas no cardiacas de riesgo bajo e intermedio: revisión sistemática y análisis costo-efectividad

\begin{tabular}{|c|c|c|c|c|c|c|c|c|c|}
\hline Autor & Año & País & Diseño & $\mathrm{N}^{*}$ & $\mathrm{ASA}^{* *}$ & $\begin{array}{l}\text { Tipo } \\
\text { de cirugía }\end{array}$ & $\begin{array}{l}\text { Análisis } \\
\text { de costos }\end{array}$ & $\begin{array}{l}\text { Estándar de } \\
\text { comparación }\end{array}$ & $\begin{array}{l}\text { Resultados / } \\
\text { Conclusiones }\end{array}$ \\
\hline $\begin{array}{r}\text { Macpherson } \\
\text { et al. }{ }^{(26)}\end{array}$ & 1990 & EE. UU. & $\begin{array}{l}\text { Cohorte } \\
\text { retrospectiva }\end{array}$ & 1109 & $N E^{* *}$ & $\begin{array}{l}\text { Electiva; } \\
\text { riesgo bajo- } \\
\text { intermedio }\end{array}$ & No & Ninguno & $\begin{array}{l}\text { La mayoria en EPO anormales eran } \\
\text { predecibles por la historia clínica. }\end{array}$ \\
\hline
\end{tabular}

ASA*: Physical status classification of the American Society of Anesthesiologists.

$\mathrm{N}^{* *}$ : número de pacientes o historias clínicas analizadas.

$\mathrm{NE}^{* * *}$ : no especificado por el autor.

ASA Guidelines: Practice advisory for preanesthesia evaluation: an updated report by the American Society of Anesthesiologists Task Force on Preanesthesia Evaluation; ACP: American College of Physicians; ACC/AHA: American College of Cardiology/American Heart Association; ESC/ESA: European Society of Cardiology (ESC) and the European Society of Anesthesiology (ESA) Guidelines.

De los datos del estudio de León-Jiménez et al. ${ }^{(12)}$ obtuvimos la tabla 2 que muestra los EPO y su costo al momento de realizar dicho estudio (2013). Con la información obtenida de la Unidad de Gestión del Paciente del Hospital Regional Lambayeque fue posible actualizar dichos costos.

Tabla 2. Exámenes preoperatorios solicitados en 100 pacientes sometidos a cirugías electivas no cardiacas de riesgo bajo e intermedio

\begin{tabular}{|lrcc|}
\begin{tabular}{|l} 
Exámenes \\
preoperatorios
\end{tabular} & N & $\begin{array}{c}\text { Costo unitario } \\
(2013)\end{array}$ & $\begin{array}{c}\text { Costo unitario } \\
(2019)\end{array}$ \\
\hline Hemoglobina & 96 & 3,2 & 4 \\
\hline Hemograma & 95 & 20,4 & 30 \\
\hline Glucosa & 98 & 15 & 18 \\
\hline Creatinina & 95 & 15 & 18 \\
\hline Urea & 95 & 15 & 18 \\
\hline Tiempo de coagulación & 93 & 4,8 & 9 \\
\hline Tiempo de sangría & 93 & 5,8 & 9 \\
\hline Grupo y factor Rh & 95 & 16 & 18 \\
\hline TP & 9 & 16 & 18 \\
\hline TTPa & 5 & 16 & 18 \\
\hline VIH & 67 & 12,5 & 30 \\
\hline Hepatitis B (HBsAg) & 66 & 28,6 & 38 \\
\hline VDRL & 57 & 6 & 7 \\
\hline Examen completo de orina & 96 & 16 & 18 \\
\hline ECG & 96 & 7,2 & 41 \\
\hline Radiografía de tórax & 1 & 23,2 & 59 \\
\hline Perfil hepático & 56 & 24,4 & 170 \\
\hline
\end{tabular}

Información del estudio de León-Jiménez et al. ${ }^{(12)}$, actualizada con datos obtenidos del HRL

León-Jiménez et al. ${ }^{(12)}$ analizaron 100 pacientes durante diez meses (octubre de 2012 a julio de 2013). De ellos, 62 fueron sometidos a colecistectomía, 16 a hernioplastia inguinal, 14 a facoemulsificación, siete a cirugía de fractura expuesta y uno a hernioplastia umbilical. No buscaron evaluar complicaciones operatorias, cambio en las decisiones terapéuticas, ni el efecto en unidades clínicas habituales o calidad de vida. Namoncura et al. (13) incluyeron a 160 pacientes sometidos a cirugías otorrinolaringológicas (adenoidectomías, amigdalectomías, punciones timpánicas, miringoplastías, timpanoplastías, rinoplastias, septoplastías, entre otras).

De acuerdo a los resultados obtenidos en este estudio (12), entre octubre 2012 a julio 2013 (10 meses), se solicitaron, en total, 1310 exámenes preoperatorios: 97 riesgos quirúrgicos (RQ) y 1213 exámenes auxiliares (laboratorio, electrocardiograma y radiografía). En promedio, se realizaron 12,1 exámenes por paciente (mínimo 7 y máximo 17) y el costo total de los EPO (RQ + exámenes auxiliares) solicitados fue 
S/ 17 363. Se realizaron 854 EPO innecesarios (promedio 8,54 por paciente: mínimo 3, máximo 15). El costo de los EPO innecesarios por cada 100 pacientes fue equivalente a S/ 8858 (S/ 10630 en un año). Al considerar el total de historias clínicas (378), los autores estimaron que el costo de los EPO innecesarios en un año, solo para estos cinco tipos de cirugías, sería aproximadamente de S/ 41856.

El costo total de los exámenes preoperatorios ( $R Q$ + exámenes auxiliares) realizados según la práctica habitual sería S/ 36094 (a los precios actuales), considerando solo las cirugías más frecuentes. El costo de los 854 EPO innecesarios sería S/ 13840 que, en un año, llegaría a ser S/ 16 608, pero estos datos solo incluyen a las 100 historias clínicas analizadas. Si se considera el número total de historias clínicas (378), el costo promedio de EPO innecesarios en un año, solo para estos cinco tipos de cirugías, sería aproximadamente S/ 62 778, al precio actual.
Para realizar el análisis costo-efectividad (ACE) utilizamos la siguiente ecuación que estima el costo-efectividad incremental (CEI) ${ }^{(24,25)}$ :

$$
\mathrm{CEI}=\frac{C_{A}-C_{B}}{E_{A}-E_{B}}
$$

La intervención "B" a evaluar sería la nueva opción o forma de solicitar exámenes preoperatorios según la guía NICE, que se compara con la intervención habitual "A" en la práctica clínica. Al combinar la información obtenida de los dos estudios previos, así como el de Souza et al. se obtiene la tabla 3. Las filas superiores de dicha tabla corresponden a los datos calculados al 2013; y las filas inferiores, al análisis con costos actualizados al 2019.

Tabla 3. Costos, resultados y análisis costo-efectividad

\begin{tabular}{|c|c|c|c|c|c|c|}
\hline Opciones & C & $\mathrm{E}$ & CEM & $\Delta C$ & $\Delta \mathrm{E}$ & CEI \\
\hline A (Rutinaria) & 17363 & $0,38^{*}-1,42^{* *} \%$ & 17363 & 8858 & $-9 \%$ & $-984,2$ \\
\hline B (NICE) & 8505 & $10 \%$ *** & 850,5 & & & \\
\hline \multicolumn{7}{|c|}{ Información con costos actualizados al 2019} \\
\hline Opciones & C & $\mathrm{E}$ & CEM & $\Delta C$ & $\Delta \mathrm{E}$ & CEI \\
\hline A (Rutinaria) & 36094 & $0,38^{*}-1,42^{* *} \%$ & 36094 & 13840 & $-9 \%$ & $-1538,8$ \\
\hline B (NICE) & 22254 & $10 \% * * *$ & 2225.4 & & & \\
\hline
\end{tabular}

* Información obtenida de Soares et al. (10,11)

** Información obtenida de Namoncura et al. (13)

***Datos obtenidos por el autor

$\mathrm{C}=$ Costo en soles por cada 100 pacientes estudiados; $\mathrm{E}=$ efectividad en porcentaje para detectar alguna alteración clínica relevante; $\mathrm{CEM}=$ costo-efectividad promedio; $\Delta \mathrm{C}$ = incremento del costo; $\Delta \mathrm{E}=$ incremento de la efectividad; $\mathrm{CEI}=$ costo-efectividad incremental.

Con base solo en las cirugías más frecuentes realizadas en el HRL, y a los precios actuales, el costo total de los exámenes preoperatorios ( $R Q$ + exámenes auxiliares) realizados según la práctica habitual sería S/ 36094 por cada 100 pacientes (Tabla 3). El costo de los 854 EPO innecesarios sería S/ 13840 que, en un año, llegaría a ser S/ 16 608. Pero estos datos solo incluyen a las 100 historias clínicas analizadas. Si se considera el número total de historias clínicas (378), el costo promedio de EPO innecesarios en un año, solo para estos cinco tipos de cirugías (colecistectomía, hernioplastia inguinal, hernioplastia umbilical, facoemulsificación, corrección de fractura expuesta), sería aproximadamente S/ 62778 al precio actual. Con la información de costos actualizada al 2019, si se dejan de solicitar EPO de manera indiscriminada y se siguen las pautas establecidas en la Guía NICE, sería posible reducir el costo de los EPO en $38,34 \%$, o lo que es lo mismo, en S/ 138,4 por cada paciente. Otros autores han descrito resultados similares. Fischer reportó que es posible lograr una reducción de costos hospitalarios de hasta $59,3 \%$ o 112 dólares por paciente sometido a una cirugía electiva, siguiendo un enfoque más conservador, en el cual los exámenes son solicitados por el anestesiólogo y no por el cirujano ${ }^{(14)}$.

El costo-efectividad incremental (CEI), con precios actualizados al 2019, es $-1538,8$, lo que significa que el costo adicional que la estrategia B (según la guía NICE) impondrá sobre la práctica habitual es de S/ -1538. Es decir, poner en práctica la nueva intervención B (solicitar EPO solo según lo recomendado por la guía NICE) representaría un ahorro de S/ 1538 (Tabla 3). 
Exámenes preoperatorios en cirugías electivas no cardiacas de riesgo bajo e

intermedio: revisión sistemática y análisis costo-efectividad

A partir de los estudios analizados, los datos obtenidos son compatibles con las conclusiones de otros trabajos ${ }^{(9-21)}$. Por lo tanto, sería adecuado realizar algunos procedimientos quirúrgicos de bajo riesgo incluso sin ningún $\mathrm{EPO}$. Una historia clínica prolija y un examen clínico exhaustivo y minucioso suelen ser suficientes para reducir significativamente el número de EPO rutinarios ${ }^{(9-31)}$. Si solo se solicitaran los EPO estrictamente necesarios, los costos por cada paciente se reducirían de manera notable, se descongestionarían los servicios de laboratorio y se reducirían los tiempos preoperatorios ${ }^{(24-31)}$.

Un estudio realizado en Brasil reportó que se realizaron un total de 3646 EPO en 800 pacientes sometidos a cirugías electivas. El 2,25\% de estos exámenes tuvieron alguna alteración, pero solo generaron un cambio de actitud en el $0,38 \%$ de pacientes ${ }^{(10,11)}$.

Según Namoncura et al. ${ }^{(13)}$, desde el punto de vista de la evaluación económica, se requieren 70 EPO rutinarios para encontrar una alteración clínicamente relevante que motive un cambio de actitud en relación al manejo del paciente. Sin embargo, ningún resultado anormal de EPO se asoció con complicaciones perioperatorias. Por el contrario, entre los casos considerados al inicio como "sin mayor riesgo" debido a que los EPO fueron normales ocurrieron complicaciones perioperatorias (sangrados posoperatorios) en el 2,9\% de casos. Es decir, los EPO rutinarios no predicen el riesgo de complicaciones, pero sí se asocian significativamente a un aumento en el número de consultas médicas, del tiempo de estancia hospitalaria, de repeticiones de exámenes y de interconsultas.

En una cohorte de más de 1000 pacientes sin factores de riesgos clínicos, Narr et al. demostraron que, a través de una evaluación concienzuda de la historia clínica y del examen físico, es totalmente factible realizar cirugías y procedimientos anestésicos de manera segura, sin aumentar el riesgo perioperatorio y sin realizar ningún EPO en los tres meses previos a la cirugía o al procedimiento ${ }^{(15)}$. Una conclusión similar fue replicada por Macpherson et al. ${ }^{(26)}$.

Los autores citados analizaron casi 3800 pacientes adultos saludables y asintomáticos sometidos a cirugías electivas. Reportaron que solamente 160 pacientes tuvieron algún EPO con resultado anormal, y de estos, 30 se pudieron haber previsto con la base de la historia clínica y el examen físico. Los resultados anormales requirieron evaluación mediante exámenes adicionales en 47 pacientes. Sin embargo, ningún EPO cambió el desenlace de la cirugía o procedimiento: ninguna cirugía se retrasó, y tampoco encontraron una asociación entre un resultado adverso y un resultado de EPO alterado. Como corolario de este y otros estudios, desde hace casi 30 años, en la Clínica Mayo no realizan EPO como tamizaje para pacientes saludables ${ }^{(16)}$.

De manera similar, es muy poca la evidencia que sustente la medición rutinaria de la glucemia basal, examen de orina, pruebas de coagulación, radiografías torácicas y electrocardiogramas para cirugías de bajo riesgo. Por lo tanto, no realizar sistemáticamente estas pruebas es una estrategia costo efectiva ${ }^{(22,32-35)}$.

\section{CONCLUSIONES}

Es claro que en los pacientes saludables y sin factores de riesgo los resultados de los EPO no afectan significativamente la conducta quirúrgica o la evolución posoperatoria, pero sí ocurre en los procedimientos electivos de riesgo bajo o intermedio. Además, unos valores normales en los resultados de EPO tampoco garantizan que el paciente no tendrá complicaciones perioperatorias. El análisis costo-efectividad realizado sustenta que, desde el punto de vista económico, no se debe realizar EPO de manera sistemática y rutinaria. La evaluación médica preoperatoria minuciosa podría reducir de manera segura el uso de EPO para este tipo de cirugías.

Contribuciones del autor: EDMR es el único responsable de la concepción, redacción, revisión y aprobación final del manuscrito.

Fuentes de financiamiento: Este artículo fue financiado por el autor.

Conflictos de interés: El autor declara no tener ningún conflicto de intereses.

\section{REFERENCIAS BIBLIOGRÁFICAS}

1. Edwards AF, Forest DJ. Preoperative laboratory testing. Anesthesiol Clin. 2018; 36(4): 493-507.

2. Smetana GW, Macpherson DS. The case against routine preoperative laboratory testing. Med Clin N Am. 2003; 87: 7-40.

3. Ibarra P. ¿Cuáles exámenes de laboratorio preanestésicos se necesitan en pacientes asintomáticos?. Rev Colomb Anestesiol. 2007; 35(4): 301-12.

4. Turnbull JM, Buck C. The value of preoperative screening investigations in otherwise healthy individuals. Arch Intern Med. 1987; 147: 1101-5.

5. Johnson RK, Mortimer AJ. Routine pre-operative blood testing: is it necessary?. Anaesthesia. 2002; 57: 914-7.

6. Contreras M. Evaluación médica preoperatoria como predictor de riesgo quirúrgico y no quirúrgico. Comunidad y Salud. 2010; 8(1): 83-96.

7. Rojas-Rivera W. Evaluación de los exámenes preoperatorios. Acta Méd Costarric. 2006; 48(4): 208-11.

8. Duddy C, Wong G. Explaining variations in test ordering in primary care: protocol for a realist review. BMJ Open. 2018; 8(9): e023117.

9. Ajimura FY, Maia ASSF, Hachlya A, Watanabe AS. Preoperative laboratory evaluation of patients aged over 40 years undergoing elective non cardiac surgery. São Paulo Med J. 2005; 123: 50-3.

10. Soares DS, Ribeiro BR, Nogueira MR, Fernandes de Azevedo VL, Vieira FA, Santana TE. Relevancia de los exámenes de rutina en pacientes de bajo riesgo sometidos a cirugías menores. Rev Bras Anestesiol. 2013; 63(2): 197-201. 
11. Rajaram N, Karim HMR, Prakash A, Sahoo SK, Dhar M, Narayan A. Prevalence and impact of abnormal routine pre-operative test results among elective surgical patients with or without co-morbidity: an observational comparative study. Niger Postgrad Med J. 2018; 25(2): 121-5.

12. León-Jiménez F, Florián-Romero E, Caján-Lontop Y, Ventura-Sandoval L, Flores-Tucto M, Gastelo-Dávila A. Exámenes prequirúrgicos innecesarios y su costo en un hospital de Lambayeque. Acta Med Per. 2014: 31(2): 90-4.

13. Namoncura PC, Breinbauer KH. Utilidad y costo-efectividad de los exámenes preoperatorios en cirugía otorrinolaringológica. Rev Otorrinolaringol Cir Cabeza Cuello. 2009; 69: 117-24.

14. Fischer SP. Cost-effective preoperative evaluation and testing. Chest. 1999; 115: 96-100.

15. Narr BJ, Warner ME, Schroeder SR, Warner MA. Outcomes of patients with no laboratory assessment before anesthesia and a surgical procedure. Mayo Clin Proc. 1997; 72: 505-9.

16. Narr BJ, Hansen TR, Warner MA. Preoperative laboratory screening in healthy Mayo patients: cost-effective elimination of tests and unchanged outcomes. Mayo Clin Proc. 1991; 66: 155-9.

17. Li Wong JC. Costo efectividad en imagenología para el diagnóstico de enfermedad periodontal Centro Imaginológico Hanny X 2016 [Tesis]. Lima: Universidad San Martín de Porres. Facultad de Medicina Humana; 2017.

18. Guttikonda N, Nileshwar A, Rao M, Sushma TK. Preoperative laboratory testing-comparison of National Institute of Clinical Excellence guidelines with current practice -an observational study. J Anaesthesiol Clin Pharmacol. 2019; 35(2): 227-30.

19. Keshavan VH, Swamy CM. Pre-operative laboratory testing: a prospective study on comparison and cost analysis. Indian J Anaesth. 2016; 60(11): 838-42.

20. Karim HMR, Prakash A, Sahoo SK, Narayan A, Vijayan V. Abnormal routine pre-operative test results and their impact on anaesthetic management: An observational study. Indian J Anaesth. 2018; 62(1): 23-28.

21. Beloeil H, Ruchard D, Drewniak N, Molliex S. Overuse of preoperative laboratory coagulation testing and $\mathrm{ABO}$ blood typing: a french national study. Br J Anaesth. 2017; 119(6): 1186-93.

22. García-Miguel FJ, García Caballero J, Gómez de Caso-Canto JA. Indicaciones de la radiografía de tórax para la valoración preoperatoria en cirugía programada. Rev Esp Anestesiol Reanim. 2002; 49: 80-8.

23. García-Miguel FJ, García Caballero J, Gómez de Caso-Canto JA. Indications for electrocardiogram in the preoperative assessment for programmed surgery. Rev Esp Anestesiol Reanim. 2002; 49: 5-12.

24. Prieto L, Sacristána JA, Antoñanzas F, Rubio-Terrés C, Pintod JL, Rovirae J. Análisis coste-efectividad en la evaluación económica de intervenciones sanitarias. Med Clin (Barc). 2004; 122(13): 505-10.

25. Campillo-Artero C, Ortún V. El análisis de coste-efectividad: por qué y cómo. Rev Esp Cardiol. 2016; 69(4): 370-3.

26. Macpherson DS, Snow R, Lofgren RP. Preoperative screening: value of preoperative test. Ann Intern Med. 1990; 113: 969-73.

27. Roizen MF. Cost-effective preoperative laboratory testing. JAMA. 1994; 271: 319-20.

28. Balk EM, Earley A, Hadar N, Shah N, Trikalinos TA. Benefits and harms of routine preoperative testing: comparative effectiveness. Rockville (MD): Agency for Healthcare Research and Quality (US); 2014. Report No. 14.

29. Ranasinghe P, Perera YS, Senaratne JA, Abayadeera A. Preoperative testing in elective surgery: Is it really cost effective?. Anesth Essays Res. 2011; 5(1): 28-32.

30. Czoski-Murray C1, Lloyd Jones M, McCabe C, Claxton K, Oluboyede $\mathrm{Y}$, Roberts $\mathrm{J}$, et al. What is the value of routinely testing full blood count, electrolytes and urea, and pulmonary function tests before elective surgery in patients with no apparent clinical indication and in subgroups of patients with common comorbidities: a systematic review of the clinical and cost-effective literature. Health Technol Assess. 2012; 16(50): 1-159.

31. Morales-Orozco C, Mata-Miranda MP, Cárdenas-Lailson LE. Costobeneficio de los exámenes preoperatorios de rutina en cirugía electiva. Cir Cir. 2005; 73(1): 25-30.

32. National Guideline Centre (UK). Preoperative tests (update): routine preoperative tests for elective surgery. London: National Institute for Health and Care Excellence (UK); 2016.

33. Feely MA, Collins CS, Daniels PR, Kebede EB, Jatoi A, Mauck KF. Preoperative testing before noncardiac surgery: guidelines and recommendations. Am Fam Physician. 2013; 87(6): 414-8.

34. Sociedad Argentina de Cardiología. Consenso de evaluación del riesgo cardiovascular en cirugía no cardiaca. Rev Argent Cardiol. 2005; 73(5): 381-96.

35. American Society of Anesthesiologists Task Force on Preanesthesia Evaluation. Practice advisory for preanesthesia evaluation: <n updated report by the American Society of Anesthesiologists Task Force on Preanesthesia Evaluation. Anesthesiology. 2012; 116: 522-38.

36. Karim MR, Yunus M, Bhattacharyya P. An observational cohort study on pre-operative investigations and referrals: how far are we following recommendations?. Indian J Anaesth. 2016; 60(8): 552-9.

37. Leal-Mateos M, Madrigal-Solís JB. Analysis of preoperative test screening in patients undergoing elective surgery at the Gynecology Department of the "Rafael Angel Calderón Guardia" Hospital during 2009. Acta Méd Costarric. 2010; 52(3): 154-8.

38. Chung F, Yuan H, Yin L, Vairavanathan S, Wong DT. Elimination of preoperative testing in ambulatory surgery. Anesth Analg. 2009; 108(2): 467-75.

39. Schein OD, Katz J, Bass EB, Tielsch JM, Lubomski LH, Feldman MA, et al. The value of routine preoperative medical testing before cataract surgery. Study of Medical Testing for Cataract Surgery. N Engl J Med. 2000; 342(3): 168-75.

40. Benarroch-Gampel J, Sheffield KM, Duncan CB, Brown KM, Han Y, Townsend CM Jr, et al. Preoperative laboratory testing in patients undergoing elective, low-risk ambulatory surgery. Ann Surg. 2012; 256(3): 518-28

\section{Correspondencia:}

Edinson Dante Meregildo Rodriguez

Dirección: Residencial Puertas del Sol, Calle 09, Mz. Y, Lt. 11; Cód. Postal 14007; Chiclayo. Lambayeque, Perú. Teléfono: +51944226126

Correo electrónico: dante_meregildo@hotmail.com

Recibido: 30 de noviembre de 2019 Evaluado: 25 de febrero de 2020 Aprobado: 25 de junio de 2020

( ) La revista. Publicado por Universidad de San Martín de Porres, Perú. (c) BY Licencia de Creative Commons Artículo en acceso abierto bajo términos de Licencia Creative Commons Atribución 4.0 Internacional. (http://creativecommons.org/licenses/by/4.0/)

ORCID iDs

Edinson Dante Meregildo Rodriguez 단 https://orcid.org/0000-0003-1814-5593 